\title{
REVIEW
}

Open Access

\section{Effectiveness of physical activity interventions for overweight and obesity during pregnancy: a systematic review of the content of behaviour change interventions}

Caragh Flannery ${ }^{1 *}$, Milou Fredrix ${ }^{2}$, Ellinor K. Olander ${ }^{3}$, Fionnuala M. McAuliffe ${ }^{4}$, Molly Byrne ${ }^{2}$ and Patricia M. Kearney ${ }^{1}$

\begin{abstract}
Background: Behaviour change techniques (BCTs) employed within PA intervention for pregnant women with a healthy body mass index (BMI) have been previously identified, however, these BCTS may differ for other weight profiles during pregnancy. The aim of this current review was to identify and summarise the evidence for effectiveness of PA interventions on PA levels for pregnant women with overweight and obesity, with an emphasis on the BCTs employed.

Methods: A systematic review and meta-analysis of PA intervention studies using the PRISMA statement was conducted. Searches were conducted of eight databases in January 2019. Strict inclusion/exclusion criteria were employed. The validity of each included study was assessed using the Cochrane Collaboration's tool for assessing risk of bias. The primary outcome measure was change in PA levels, subjectively or objectively measured, with physical fitness as a secondary outcome. All intervention descriptions were double coded by two authors using Michie's et al's BCT taxonomy V1. Meta-analyses using random effect models assessed the intervention effects on PA. Other PA outcomes were summarised in a narrative synthesis.
\end{abstract}

Results: From 8389 studies, 19 met the inclusion criteria 13 of which were suitable for inclusion in a meta-analysis. The remaining 6 studies were described narratively due to insufficient data and different outcome measures reported. In the meta-analysis, comparing interventions to a control group, significant increases were found in the intervention group for metabolic equivalent (SMD $0.39[0.14,0.64], Z=3.08 P=0.002)$ and physical fitness $\left(\mathrm{VO}_{2}\right.$ max) $(\mathrm{SMD} 0.55[0.34$, $0.75], Z=5.20 P=<0.001)$. Of the other six, five studies reported an increase in PA for the intervention group versus the control with the other study reporting a significant decrease for women in their 3rd trimester $(p=0.002)$. 'Selfmonitoring of behaviour' was the most frequently used BCTs (76.5\%), with 'social support' being newly identified for this pregnant population with overweight or obesity.

Conclusions: This review identified a slight increase in PA for pregnant women with overweight and obesity participating in interventions. However, due to the high risk of bias of the included studies, the results should be interpreted with caution. PA measures should be carefully selected so that studies can be meaningfully compared and standardised taxonomies should be used so that BCTs can be accurately assessed.

Keywords: Physical activity, Pregnancy, BMI, Intervention, Behaviour change, Behaviour change techniques, Systematic review

\footnotetext{
* Correspondence: cflannery@ucc.ie

${ }^{1}$ School of Public Health, University College Cork, Cork, Ireland

Full list of author information is available at the end of the article
}

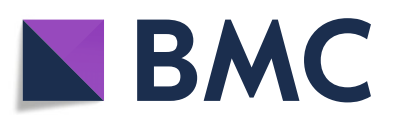

(c) The Author(s). 2019 Open Access This article is distributed under the terms of the Creative Commons Attribution 4.0 International License (http://creativecommons.org/licenses/by/4.0/), which permits unrestricted use, distribution, and reproduction in any medium, provided you give appropriate credit to the original author(s) and the source, provide a link to the Creative Commons license, and indicate if changes were made. The Creative Commons Public Domain Dedication waiver (http://creativecommons.org/publicdomain/zero/1.0/) applies to the data made available in this article, unless otherwise stated. 


\section{Background}

Overweight and obesity during pregnancy is an increasing public health concern. Overweight is defined as BMI $\geq 25 \mathrm{~kg} / \mathrm{m}^{2}$ and obesity is defined as a BMI $\geq 30 \mathrm{~kg} / \mathrm{m}^{2}$ which is assessed at the first antenatal consultation [1]. Overweight and obesity is associated with a number of adverse maternal and neonatal outcomes including increased rates of gestational diabetes mellitus (GDM), pre-eclampsia, caesarean section, instrumental delivery and preterm delivery $[2,3]$. Additionally excessive gestational weight gain is associated with weight retention and type 2 diabetes in the longer term $[4,5]$.

Physical activity has been identified as a modifiable lifestyle factor that could help prevent pregnancy complications, help with weight management and reduce the risk of GDM for women with overweight and obesity [6]. Previous research has found that physically active pregnant women report better health than less physically active women as well as an increase in functional ability and a reduction in nausea, fatigue and stress [7-9]. Despite the significant health benefits, based on self-report, women tend to be less active in pregnancy due to fatigue and discomfort $[10,11]$. International guidelines recommend 30 min of daily moderate intensity physical activity for pregnant women [12-15]. A review which updated the latest evidence concerning exercise during pregnancy found that in the United States only $15.8 \%$ of women engaged in exercise during pregnancy [16]. Similarly, low levels of physical activity have been reported in an Irish cohort of pregnant woman with only $21.5 \%$ of women meeting the current recommendations $[9,11]$. Furthermore, a study examining lifestyle changes using the Pregnancy Risk Assessment Monitoring system (PRAMS) in Ireland found that adherence to physical activity guidelines of moderate intensity activity was low (12.3\%) but was particularly low for pregnant women with overweight and obesity (6.4\%) [17]. Therefore, pregnant women with overweight and obesity should be encouraged to follow an exercise programme in order to get the best health outcomes for both mother and baby [18].

Behavioural change is complex and involves identifying effective and efficient techniques to bring about change [19]. These techniques are called behaviour change techniques (BCTs) and are defined as 'an active component of an intervention designed to change behaviour' pg. 145 [20]. In order to identify the intervention content or behavioural component of an intervention, the BCT taxonomy V1 was developed [20]. The BCT Taxonomy V1 consisting of 93 different BCTs (16 categories) is a useful tool to extract the active components of successful and unsuccessful behaviour change interventions.

However, reviews of lifestyle interventions during pregnancy are varied and results to date are conflicting
[21-23]. Many of the interventions promoting lifestyle changes throughout pregnancy are multidimensional incorporating a combination of diet and physical activity $[2,22,24,25]$. These interventions tend to focus on medical or obstetric outcomes such as reducing excessive gestational weight gain (GWG) or GDM with less focus on the behavioural outcomes such as physical activity.

According to a review by Currie et al. (2013) which evaluated the content of physical activity interventions in pregnancy, interventions within the review were most effective when BCTs were employed and delivered face to face [26]. However, there is uncertainty around which underlying BCTs are most effective. Collins et al. suggested two components that need to be explored in order to identify effective interventions. These are intervention programme (employed BCTs) and intervention delivery (intervention provider, format, setting, recipient, intensity, duration and fidelity of the intervention) [27]. A review examining behaviour-change interventions for obese adults with additional risk factors or comorbidities found suggestive evidence for an association between greater numbers of BCTs and greater weight loss [28]. Furthermore, a review examining intervention features of dietary and physical activity interventions for patients with type 2 diabetes revealed BCTs associated with clinically significant reductions in $\mathrm{HbA}_{1 \mathrm{c}}$ [29]. Previous systematic reviews in the area of pregnancy [26, 30] have assessed intervention effectiveness including GWG [21-23, 31, 32] and GDM [33] but have not examined the intervention programme content itself.

BCTs have been retrospectively identified in a number of systematic reviews [24, 34]. The identification of optimal BCTs necessary for increasing physical activity in a healthy adult population found six important techniques including: providing information on the likely consequences of specific behaviour, action planning, reinforcing effort or progress, providing instructions, facilitative social comparison and time management [24]. However, the techniques associated with increasing physical activity for adults with obesity were different, using BCTs such as 'teach to use prompts/cues', 'prompt practice' or 'prompt rewards' instead. Thus, to develop effective physical activity interventions it may be important to consider tailoring intervention techniques to the target population [35]. The significance of BCTs may be different for pregnant women compared to non-pregnant women since pregnancy is a unique time where women may be more receptive to improving health behaviours [36]. In pregnancy, using the most up-to-date BCT taxonomy, Currie et al. identified the most common BCTs for healthy weight pregnant women, including 'goal setting', 'feedback and planning', 'repetition and substitution', 'shaping knowledge' and 'comparison of behaviours' [26]. Furthermore, the value of 
these techniques is likely to depend on the weight profile of the pregnant population and successful BCTs may differ for pregnant women with overweight and obesity compared to pregnant women with a healthy BMI [37-40].

Therefore, the aims of this systematic review and metaanalysis was to identify and summarise the evidence for the effectiveness of physical activity interventions for pregnant women with overweight and obesity on physical activity levels and identify which BCTs were most frequently used in these interventions and determine which were most effective in improving physical activity levels.

\section{Methods}

This systematic review and meta-analysis were reported in accordance with the Preferred Reporting Items for Systematic reviews and Meta-Analyses (PRISMA) statement [41]. The review protocol was pre-registered with the International Prospective Register of Systematic Reviews (PROSPERO) database (CRD42016033423).

\section{Eligibility criteria \\ Types of studies}

Eligible study designs included pilot randomised controlled trials, randomised control trials (RCTs), non-randomised control trials, quasi RCTs, and quasi-experimental studies of physical activity interventions, aimed at maintaining or increasing physical activity levels conducted in any setting. Furthermore, for inclusion, all interventions had to target pregnant women with overweight and obesity with a body mass index $(\mathrm{BMI}) \geq 25 \mathrm{~kg} / \mathrm{m}^{2}$, have at least one component focusing explicitly on physical activity, and include a discernible BCT in the intervention description. Control groups were classified as a comparator intervention or usual care if stated. Usual care would indicate standard antenatal care for pregnant women. Studies were included regardless of treatment intensity, duration or mode of delivery of the intervention. Only studies published in English were included. Studies published in the grey literature (non-peer reviewed or without scientific credibility) were excluded.

\section{Types of participants}

Participants included pregnant women with a prepregnancy or early pregnancy BMI $\geq 25 \mathrm{~kg} / \mathrm{m}^{2}$ and singleton pregnancies.

\section{Types of outcome(s) measures}

Studies were included that reported any of the following primary outcome measures: change in physical activity levels subjectively (e.g., self-report) or objectively measured (e.g., step count) at baseline and post intervention. Secondary outcome included studies that reported $\mathrm{VO}_{2}$ max as a measure of physical fitness.

\section{Information sources}

\section{Searches}

MEDLINE, EMBASE, PsychInfo, CINAHL, Cochrane Library, PEDro, SportDiscus and PubMed databases were searched from inception. The searches were undertaken in January 2019. The search strategy for each database is available in Additional file 1. Phrases and MESH headings for each component of the population, intervention, comparator and outcome framework (PICO), were combined using OR and then using AND (maternal, pregnancy, pregnant woman, expectant mothers; lifestyle, lifestyle modification, health promotion, behaviour change, physical activity, exercise, fitness, activities of daily living, human activities, group exercise, randomised controlled trial, intervention trials and clinical trials; standard care; physical activity, gestational weight gain and gestational diabetes). Manual searches of reference lists were conducted on all eligible articles following screening.

\section{Study selection}

One author (CF) conducted the searches and imported citations in to a reference management software package (Endnote version 7). Duplicates were removed. In the first screening stage, all titles of the search results were examined and irrelevant titles were removed if they did not meet the inclusion criteria. In the second stage, title and abstracts were screened. Ten percent of title and abstracts were double screened by authors (MB, EO, PK and FMA). Any discrepancies were resolved by consensus. Cohen's kappa $(k)$ was calculated to determine the extent of interrater agreement $[42,43]$. In the third stage of the screening process, relevant articles were obtained in full and assessed against the inclusion and study quality criteria. Full text screening was conducted by (CF) and checks were made by $2 \mathrm{~s}$ reviewers ( $\mathrm{MB}$ and $\mathrm{PK}$ ); discrepancies were resolved by consensus. The number of articles at each stage can be seen in the PRISMA flow chart (Fig. 1).

\section{Data extraction}

A data form was developed based on the Workgroup for Intervention Development and Evaluation Research (WIDER) framework for the scientific reporting of behaviour change interventions [44]. Data from each included study were extracted by one reviewer (CF) and independently checked by two others (MB and PK). In case of discrepancies, consensus was reached through discussion. Extracted data included detailed description of the interventions (study design, participant information, details of the intervention, sample size, type of contact and setting) and BCTs included in the intervention. Physical activity measures for baseline, pre and post intervention, where possible, were extracted from studies 


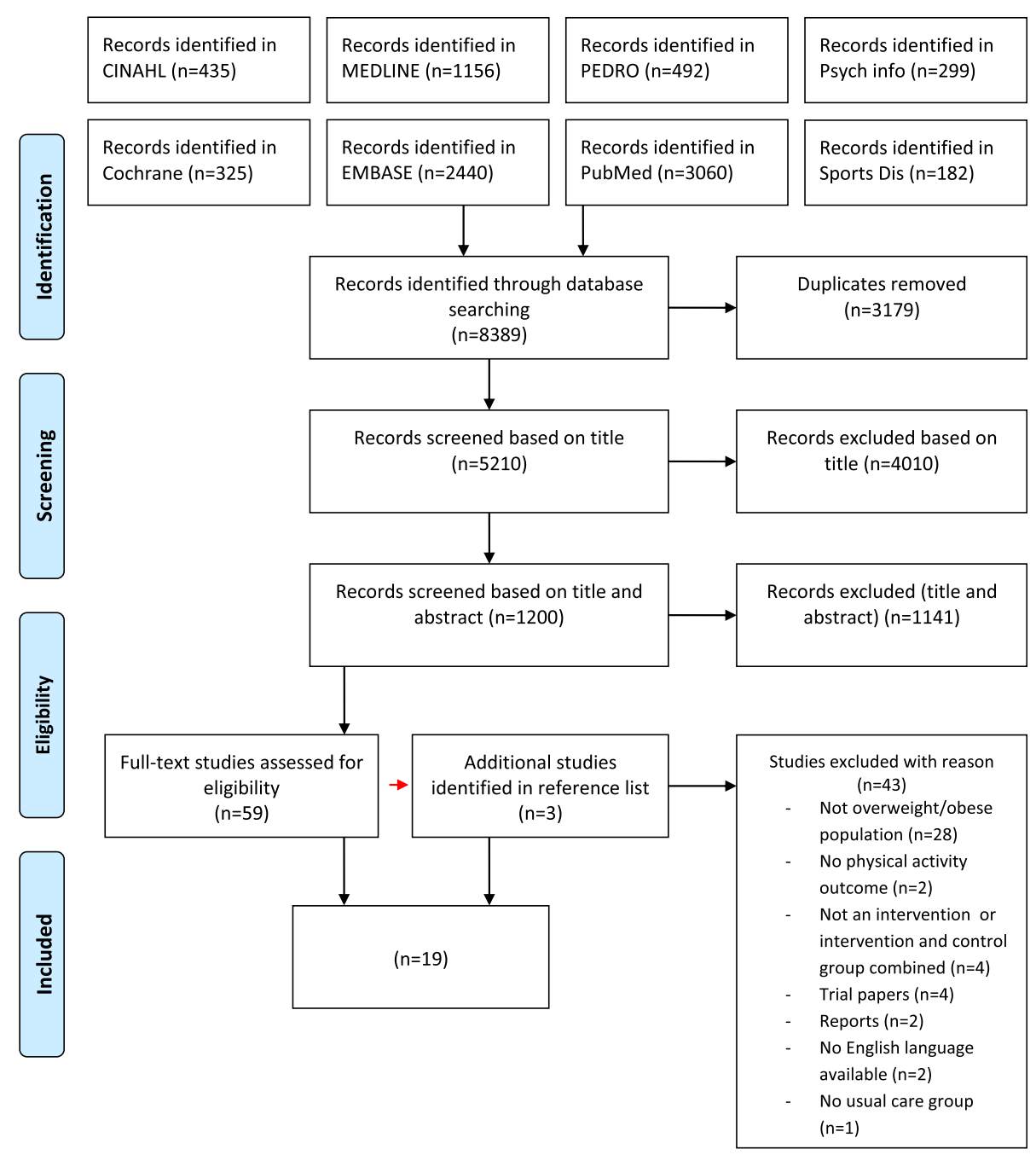

Fig. 1 PRISMA Flow Diagram

or calculated using reported means, standard deviations, and sample sizes at baseline, post-intervention.

\section{Coding of BCTs}

The BCT taxonomy V1 was used to identify the behavioural components of the intervention within each included study. This validated taxonomy consists of 93 different BCTs divided into 16 categories. A BCT was only coded when it was explicitly mentioned in the intervention or supporting materials (study protocols). The BCT coding was completed independently by two reviewers (CF and $\mathrm{MF}$ ) who underwent training in $\mathrm{BCT}$ coding using the $\mathrm{BCT}$ taxonomy. Inter-rater reliability was calculated [43] and discrepancies were discussed until $100 \%$ agreement was achieved.

\section{Risk of bias assessment}

Following the intensive screening process only RCTs were included, therefore, the validity of each included study was assessed using the Cochrane Collaboration's tool for assessing risk of bias [45]. This tool assesses key methodological domains; sequence generation, allocation concealment, blinding of participants, personnel and outcome assessors, incomplete outcome data, selective outcome reporting, other sources of bias [45]. The risk of bias was assessed by one reviewer (CF) and in the case of uncertainty consensus was reached through discussion with two authors (MB and PK).

\section{Strategy for data synthesis Effect of the intervention}

Results from the included studies were combined in a meta-analysis if sufficient outcome data were available from at least two studies. When an intervention reported data at several time points during pregnancy, the last measure before birth was used. Continuous data were summarized as mean difference and standard deviations (SD). Where possible, means and SD were calculated 
from median and interquartile range [46]. Within the meta-analysis, primary and secondary physical activity outcomes reported on the same scale (e.g. MET, Steps and $\mathrm{VO}_{2}$ max) were combined using standardised mean differences (SMD). For all effect sizes, 95\% Confidence Intervals $(\mathrm{CI})$ were used and results were pooled using a random effects model (inverse-variance approach based on weighted SMDs) using Review Manager Software (version 5.3: Review Manger). Furthermore, the $\mathrm{I}^{2}$ statistic was used to indicate the percentage of total variation [45]. If data was not available for pooling outcomes, all other physical activity outcomes measures were combined in a narrative synthesis. To test the robustness of the findings, risk of publication bias was conducted using Stata (version 13.1). Funnel plots were generated and a test for statistical significance for funnel plot asymmetry was performed using Eggers test [47].

\section{BCTs}

A BCT was only coded when there was clear evidence of its inclusion in the intervention and it was identified as present by both reviewers. The total number of BCTs was recorded and the frequency of identified BCTs was quantified. Subgroup analysis was selected as a method to examine the effectiveness of different BCTs on outcomes included in the meta-analysis. Subgroup analysis would only be conducted if a meta-analysis was conducted with 10 or more studies. Pearson's r correlation coefficient was used to investigate the relationship between the number of BCTs used and the outcome effect sizes.

\section{Results}

\section{Study selection}

Searches conducted in January 2019 found 8389 studies. Nineteen studies were included (Fig. 1), describing 3 pilot randomised controlled trials [48-50] and 16 randomised controlled trials [51-66] of which 2 were multicentre [60, 61], 2 were prospective [62,63], 2 were parallel [64, 65] and 1 was a nested randomised controlled trial [66]. Cohen's kappa $(k)$ was calculated to determine the extent of inter-rater agreement during the screening phase and a substantial agreement was reached $(k=0.63)$. The total number of participants included in all studies was 7822, ranging from 12 [56] to 1924 [60] in individual studies.

Health outcomes measured in the interventions included gestational weight gain, fasting insulin, fasting glucose, gestational diabetes, gestational age (weeks), and infant birth weight $(\mathrm{kg})$. Eight studies were investigations targeting physical activity promotion alone [48, $50,53,54,56,57,64,65]$ while 11 others were of interventions targeting diet and physical activity $[49,51,52$, $55,58-63,66]$. Fourteen studies described their control groups as receiving standard routine antenatal care.
There was no clear definition of standard antenatal care in these studies. Five studies described their control group as those who were not provided with the intervention [64], those who were not provided with physical activity recommendations or restricted from physical activity participation [50,55]. The final two studies compared the intervention with a stretching group which included relaxation (respiratory exercises and light stretching) [57] or having access to additional information from a website [59].

\section{Characteristics of included studies}

Studies were conducted in Australia [48, 56, 60, 66], the Netherlands [54], the United States of America (USA) [49, 50, 59], Brazil [53, 57], New Zealand [64], Ireland [58], the United Kingdom (UK) [61], Italy [63], Finland [52], Denmark [55, 62], Belgium [51] and Norway [65]. Twelve studies were interventions that targeted pregnant women with overweight and obesity $[49,50,53,54,57-60$, 63-66] while seven studies focused on pregnant women with obesity only $[48,51,52,55,56,61,62]$ (See Table 1).

\section{Intervention characteristics}

Intervention duration ranged between 8 and 24 weeks. An explicit theoretical basis was mentioned in 6 out of the 19 studies, including stage theories of health decision making, behavioural modification, the transtheoretical model, social cognitive theory and control theory $[49,51,58,60,61,66]$. Most of the interventions were based in clinical settings $[48,49,51,52$, $54,55,57-63,65,66]$, in the participants home [56, $64]$ or in a combination of both $[50,53]$. Interventions were mostly delivered face-to-face and or via phone contact (phone calls, smartphone application) and were commonly provided by a physiotherapist, nutritionist/dieticians, study researchers, health educators or other health care professionals. The delivery of interventions ranged from at least one face-to face contact moment to phone contact throughout the intervention. One study did not specify contact type [56]. Table 2 provide details on the intervention components and BCTs in the included studies.

\section{Risk of bias assessment}

Overall risk of bias was high. Three studies were rated as having high potential risk of bias. Nine studies inadequately reported methodological quality indicators (e.g. studies lacked information on randomisation, allocation and outcome assessment concealment and inadequate missing data handling, see Additional file 2). For most studies, there was inadequate information to make judgements about methodological quality and the risk of bias. Seven studies were rated as low risk as they provided adequate information; however, five used self-report measures for physical activity. Furthermore, overall, blinding 


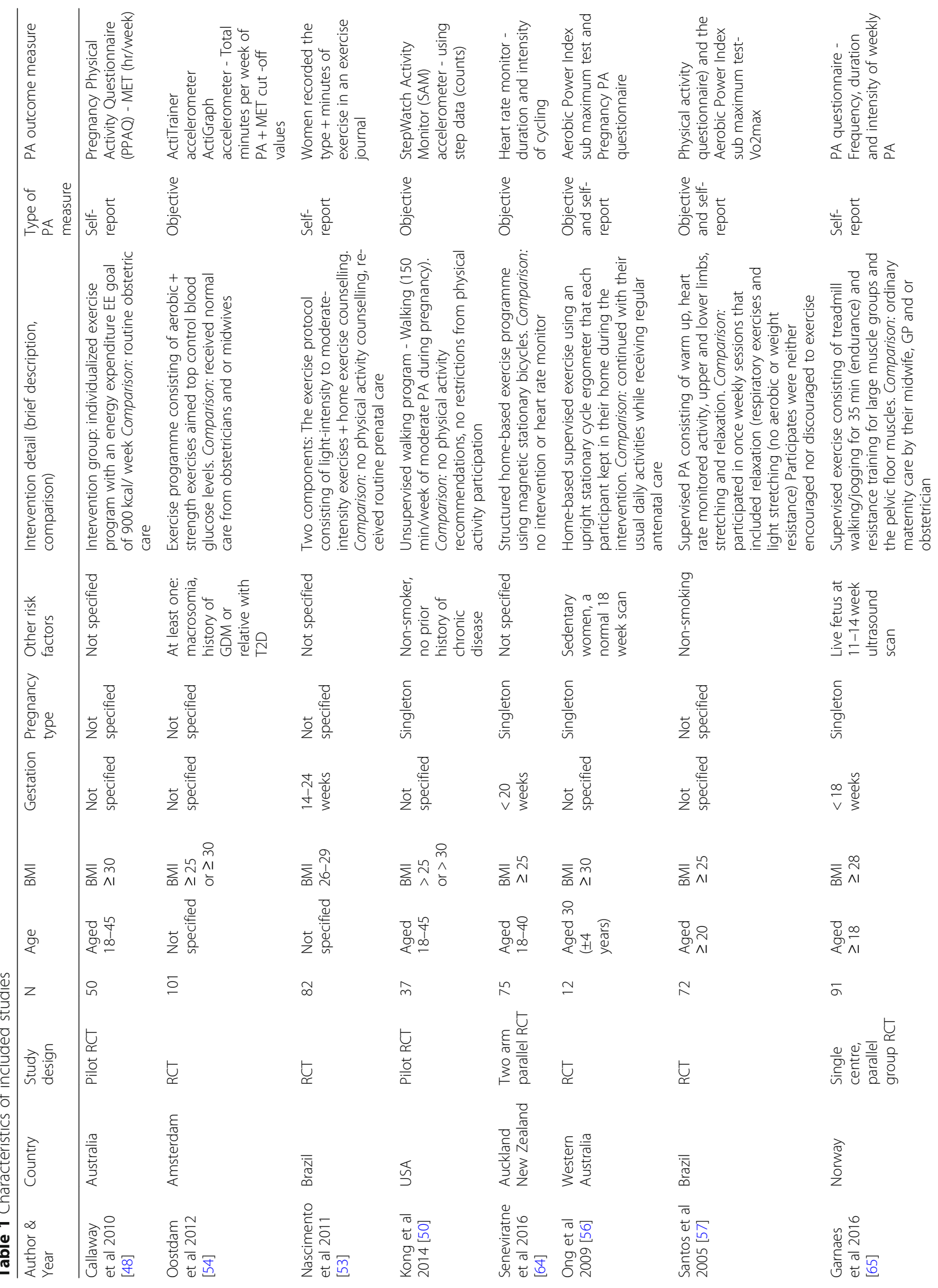




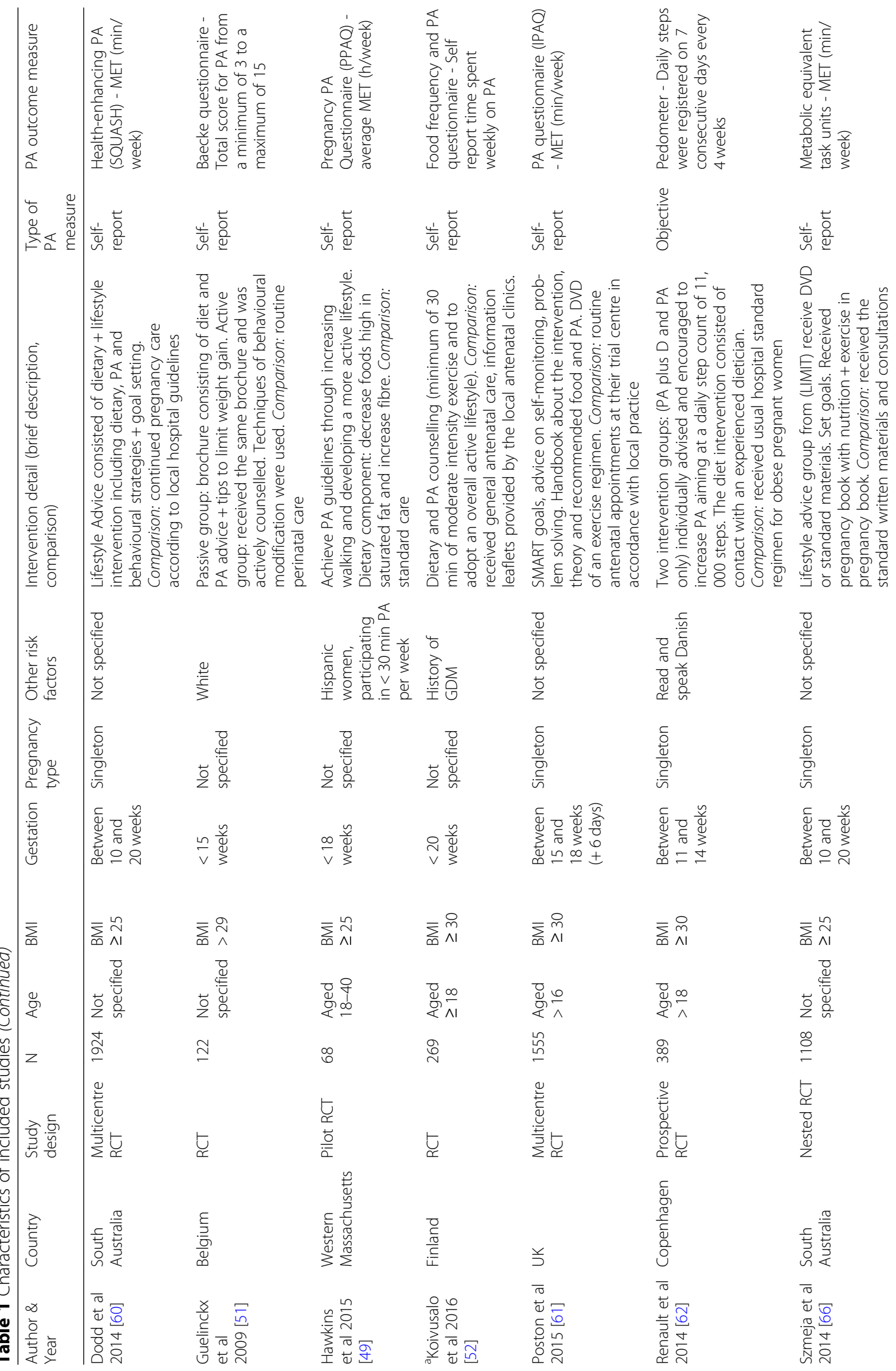




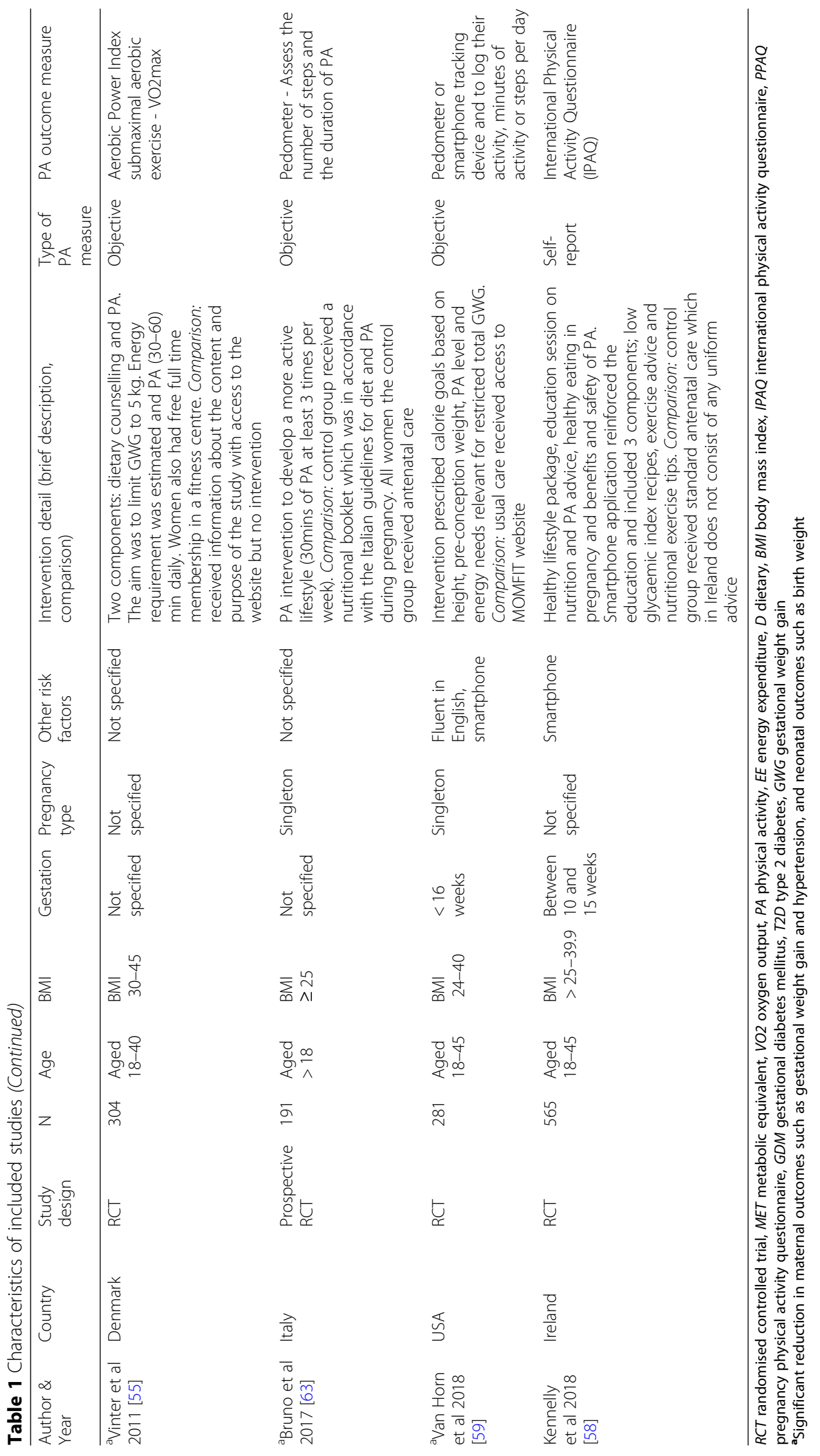




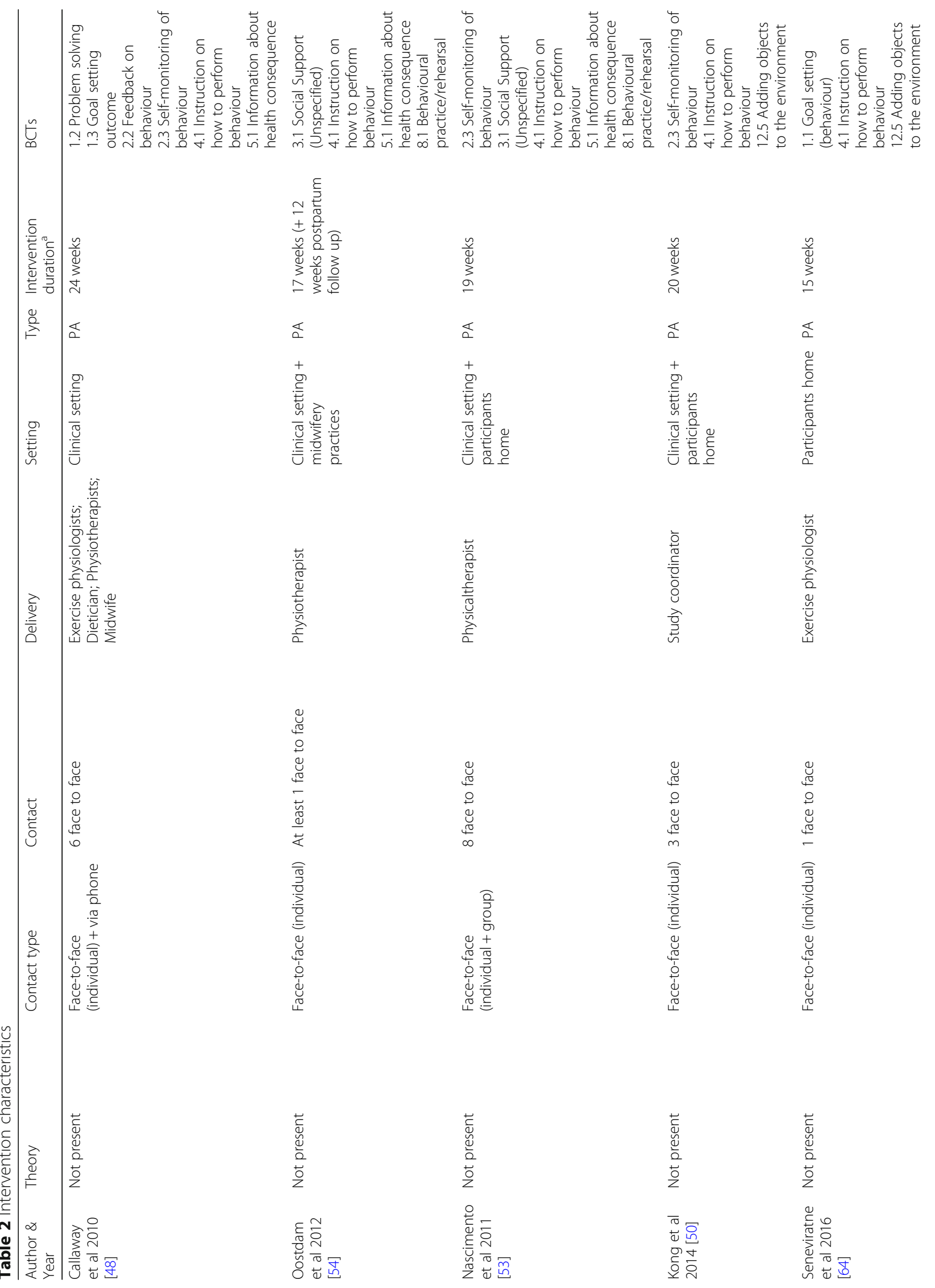




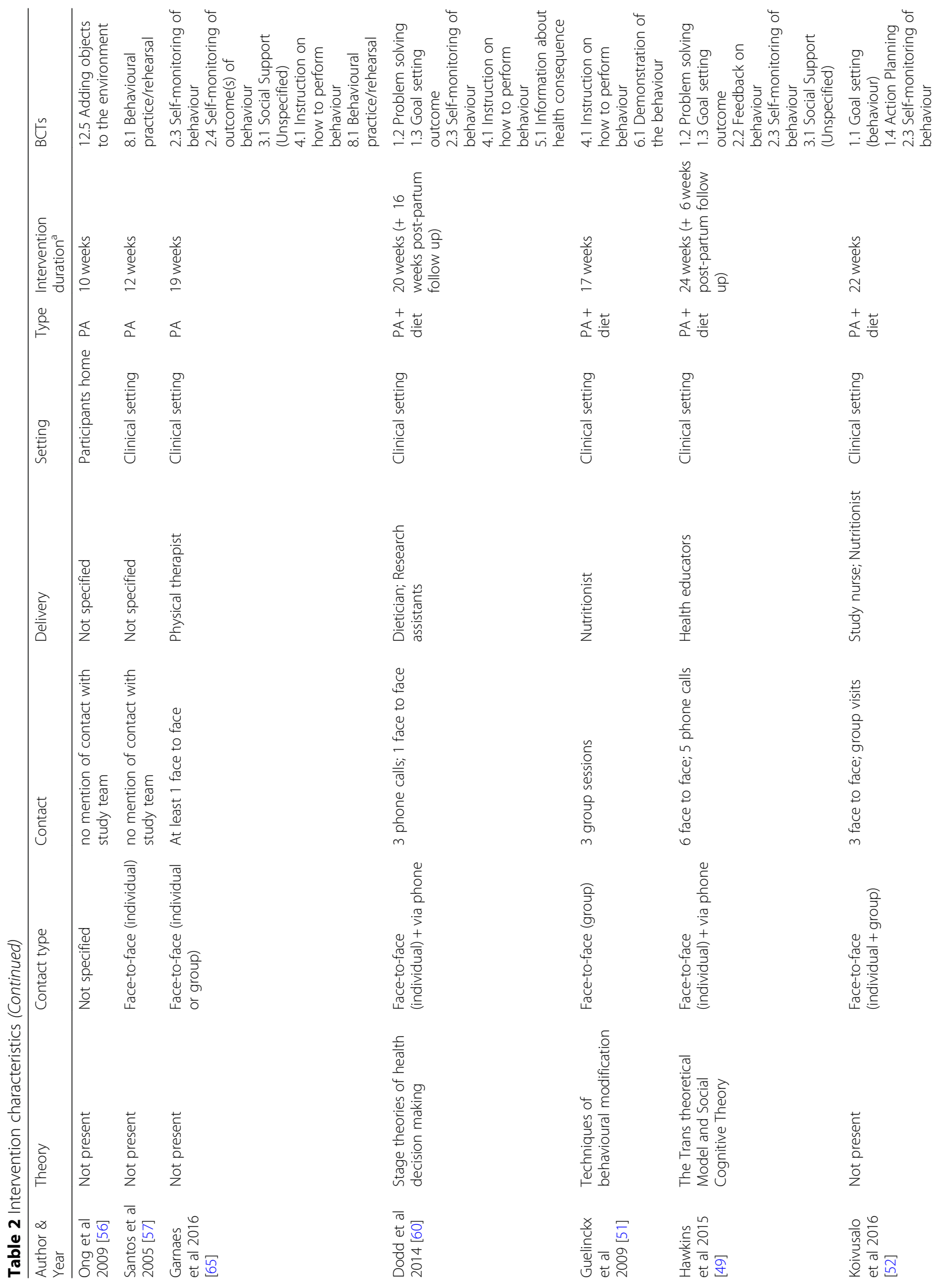




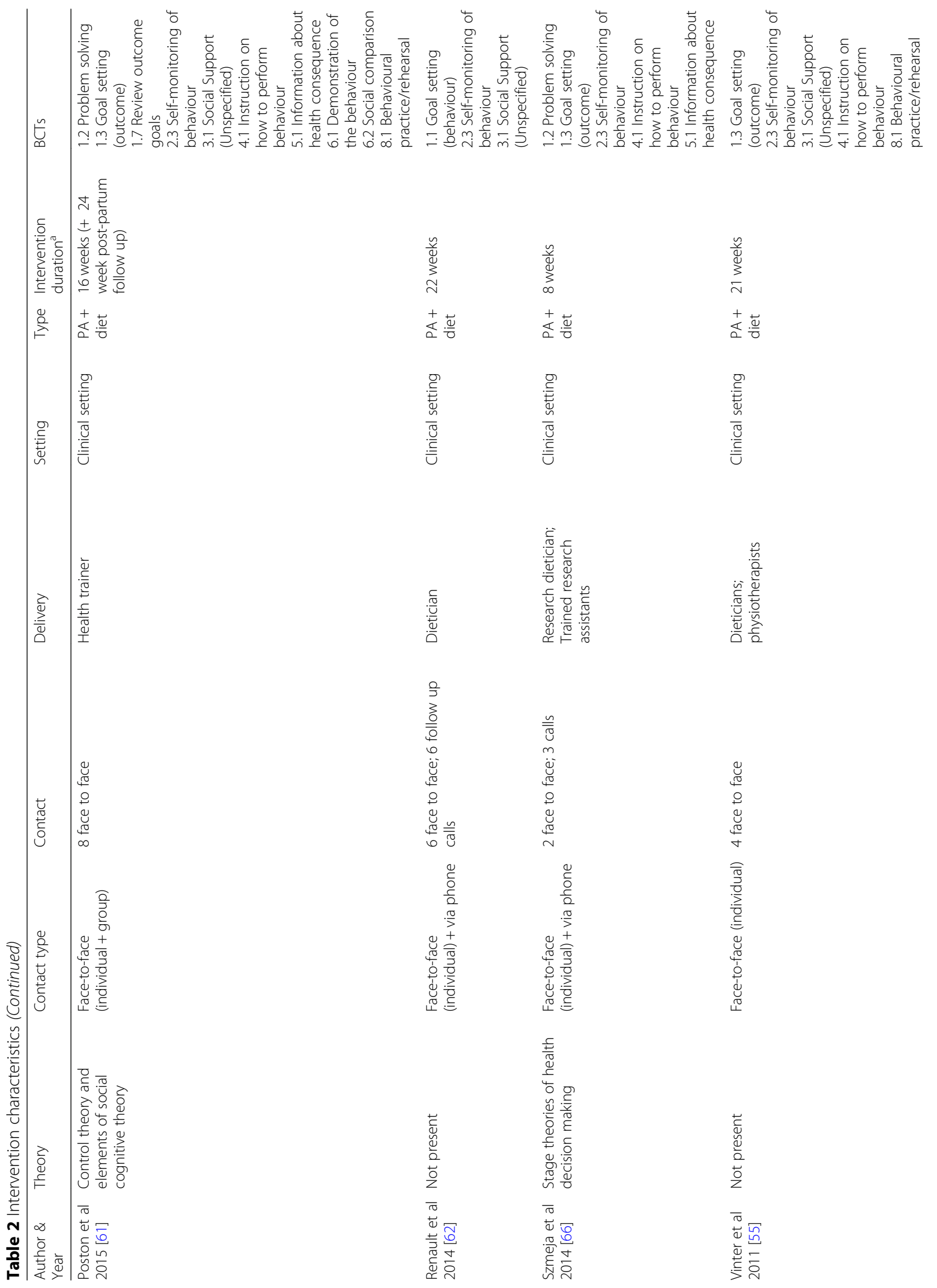




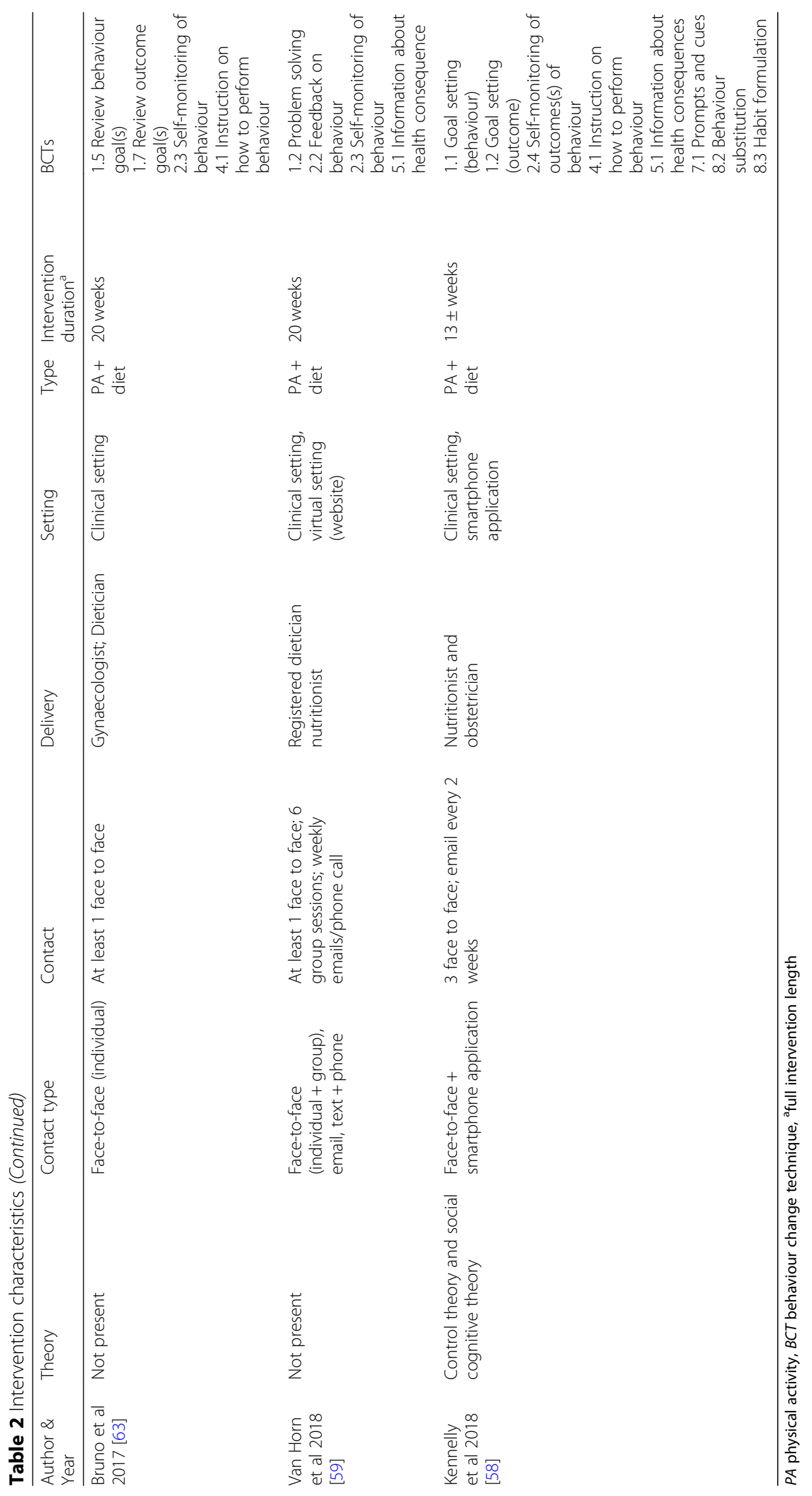




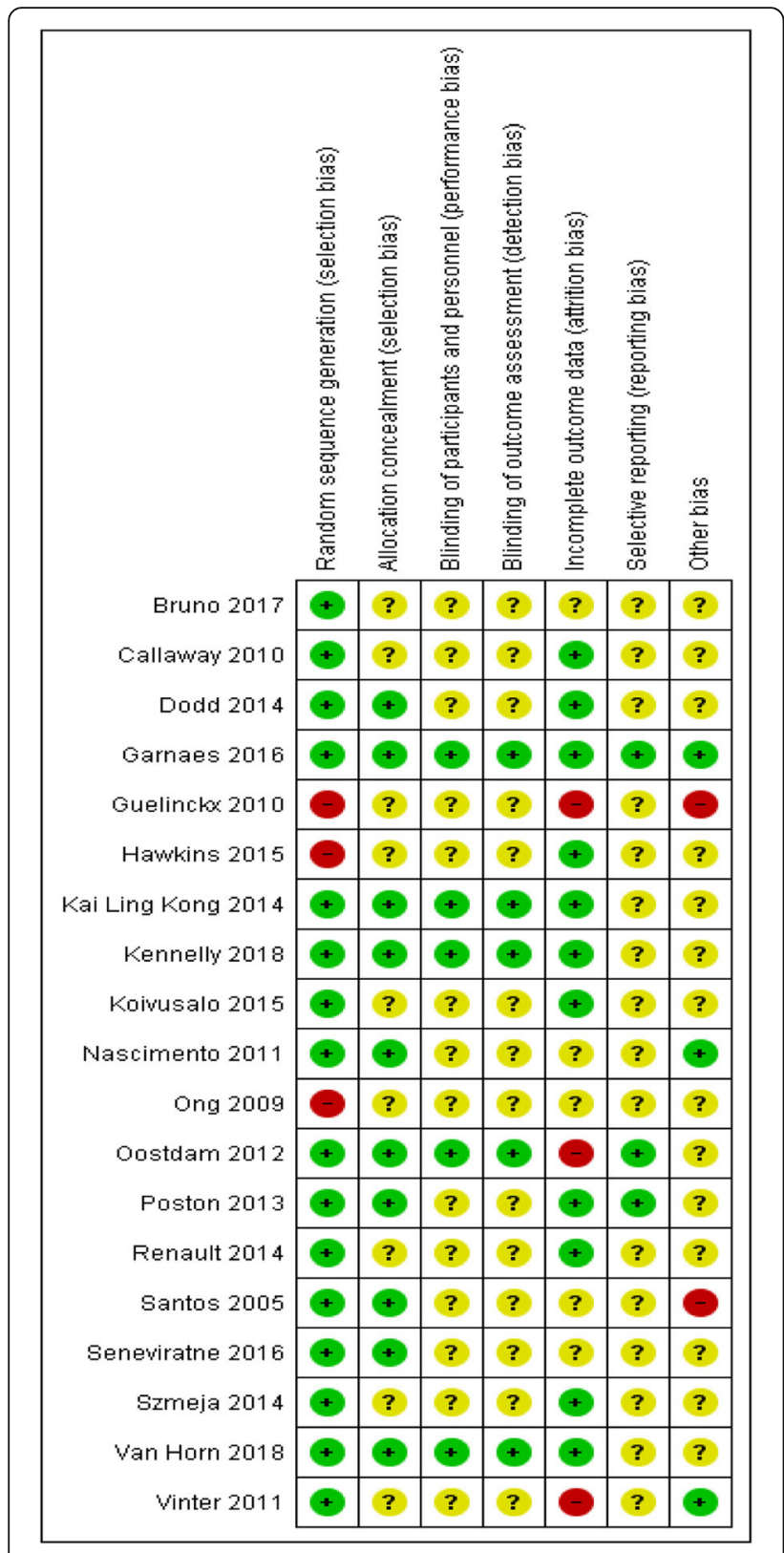

Fig. 2 Risk of Bias

(performance bias and detection bias) was considered to have the highest risk as most studies failed to document the blinding procedures. A summary of the risk of bias for all 19 studies is shown in Fig. 2 (and Additional file 2). Studies were not excluded due to high risk and /or unclear risk of bias. Instead, sensitivity analyses were carried out for MET minutes per week and for step count data (see Additional file 3) in order to assess the influence of methodological quality on effect size. Sensitivity analysis was not conducted for $\mathrm{VO}_{2}$ max due insufficient data.

\section{Publication bias}

For MET min per week, the Eggers test for bias was 2.51 [CI: $95 \%-3.08,8.11] p$-value $=0.314$ which suggests that publication bias could not be detected. The funnel plot can be seen in Additional file 4. Eggers test and funnel plots were not conducted for step count data or $\mathrm{VO}_{2}$ max as insufficient data was available.

\section{Effectiveness of the intervention Physical activity outcomes}

A wide variety of measures was used to assess physical activity in each of the included papers. Eight trials assessed physical activity objectively: four trials used pedometers deriving step-count $[50,59,62,63]$, one trial used an accelerometer to create metabolic equivalent (MET) [54], heart rate monitor data was collected to identify the duration and intensity of physical activity [64] and $\mathrm{VO}_{2}$ max was used as an indicator for physical fitness in two studies $[55,57]$. Of the 19 included papers, 13 provided data suitable for inclusion in a metaanalysis [48-50, 54, 55, 57, 60-63, 66] (Fig. 3).

\section{Primary physical activity outcomes Metabolic equivalent (MET) - minutes per week}

Physical activity expressed in METS represents the metabolic equivalent intensity levels for activities with moderate intensity activity classified as 3-5 METS. Therefore 150 min of moderate intensity physical activity is equivalent to $450-750 \mathrm{MET} /$ minutes per week [67, 68]. Eight studies comparing interventions using METs minutes per week to a control group were combined in a meta-analysis $[48,49,54,58-61,66]$. A meta-analysis using standardised mean differences at follow up demonstrated a significant increase in MET minutes per week (SMD 0.39 [0.14, 0.64], $\mathrm{Z}=3.08 P=0.002$ ). However, the studies were significantly heterogeneous $\left(x^{2}=98.65\right.$, d.f. $=$ $7[P<0.0001), \mathrm{I}^{2}=93 \%$.

\section{Step count data}

Three studies comparing physical activity interventions to a control group that used step count data at follow up were combined (Fig. 3). One of these studies included multiple intervention arms which were combined, however participants in the control group of this study did not wear pedometers so step count data was not available for comparison [62]. The studies were significantly heterogeneous $\left(\chi^{2}=6.36\right.$, d.f. $=1[P=0.01), \mathrm{I}^{2}=84 \%$ and demonstrated no significant difference in physical activity steps per day between the intervention and control groups at follow up (SMD $-0.08[-1.01,0.85], Z=0.16$ $P=0.87)$. 


\section{MET $m / w k$}

\begin{tabular}{|c|c|c|c|c|c|c|c|c|c|c|}
\hline \multirow[b]{2}{*}{ Study or Subgroup } & \multicolumn{3}{|c|}{ PA intervention group } & \multicolumn{3}{|c|}{ Control } & \multicolumn{2}{|r|}{ Std. Mean Difference } & \multirow{2}{*}{\multicolumn{2}{|c|}{$\begin{array}{l}\text { Std. Mean Difference } \\
\text { IV, Random, } 95 \% \mathrm{Cl}\end{array}$}} \\
\hline & Mean & SD & Total & Mean & SD & Total & Weight & IV, Random, $95 \% \mathrm{Cl}$ & & \\
\hline Callaway 2010 & 576 & 192 & 25 & 162 & 156 & 25 & $6.8 \%$ & $2.33[1.60,3.06]$ & & \\
\hline Dodd 2014 & 5,850 & 3,955 & 974 & 5,518 & 3,845 & 950 & $15.9 \%$ & $0.09[-0.00,0.17]$ & & $=$ \\
\hline Hawkins 2015 & $-1,308$ & 1,002 & 33 & $-1,668$ & 972 & 35 & $10.1 \%$ & $0.36[-0.12,0.84]$ & & - \\
\hline Kennelly 2018 & 639 & 436 & 165 & 469 & 328 & 170 & $14.5 \%$ & $0.44[0.22,0.66]$ & & $\pi$ \\
\hline Oostdam 2012 & 150 & 114 & 15 & 178 & 89 & 19 & $7.3 \%$ & $-0.27[-0.95,0.41]$ & & \\
\hline Poston 2013 & 2,156 & 972 & 629 & 1,694 & 786 & 786 & $15.8 \%$ & $0.53[0.42,0.64]$ & & $=$ \\
\hline Szmeja 2014 & 5,757 & 4,062 & 541 & 5,866 & 3,859 & 564 & $15.7 \%$ & $-0.03[-0.15,0.09]$ & & \\
\hline Van Horn 2018 & 228 & 121 & 126 & 180 & 101 & 124 & $13.9 \%$ & $0.43[0.18,0.68]$ & & - \\
\hline Total $(95 \% \mathrm{Cl})$ & & & 2508 & & & 2673 & $100.0 \%$ & $0.39[0.14,0.64]$ & & A \\
\hline \multicolumn{7}{|c|}{$\begin{array}{l}\text { Heterogeneity: } \text { Tau }^{2}=0.10 ; \mathrm{Chi}^{2}=98.65, \mathrm{df}=7(P<0.00001) ; I^{2}=93 \% \\
\text { Test for overall effect: } Z=3.08(P=0.002)\end{array}$} & & & $\begin{array}{cc}-2 & -1 \\
\text { Favours Control }\end{array}$ & $\begin{array}{lcl}0 & 1 & 2 \\
\text { Favours Intervention }\end{array}$ \\
\hline
\end{tabular}

\section{Steps Count Data}

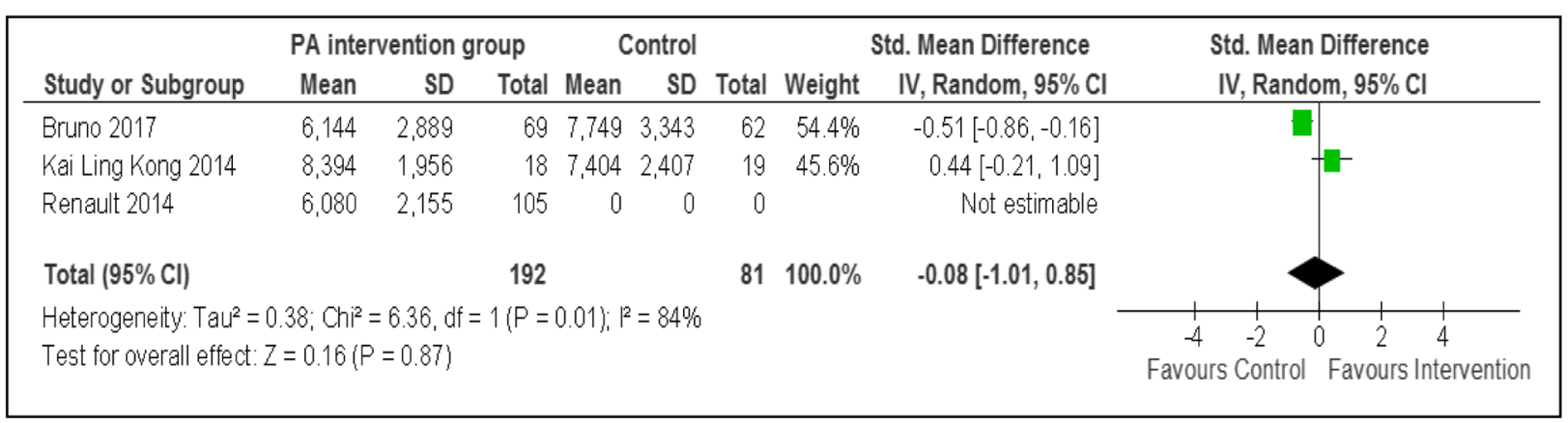

\section{$\mathrm{VO}_{2} \max$}

\begin{tabular}{|c|c|c|c|c|c|c|c|c|c|c|}
\hline \multirow[b]{2}{*}{ Study or Subgroup } & \multicolumn{3}{|c|}{ PA intervention group } & \multicolumn{3}{|c|}{ Control } & \multicolumn{2}{|r|}{ Std. Mean Difference } & \multirow{2}{*}{\multicolumn{2}{|c|}{$\begin{array}{l}\text { Std. Mean Difference } \\
\text { IV, Random, } 95 \% \mathrm{Cl}\end{array}$}} \\
\hline & Mean & SD & Total & Mean & SD & Total & Weight & IV, Random, $95 \% \mathrm{Cl}$ & & \\
\hline Santos 2005 & 23 & 2.3 & 150 & 22 & 1.6 & 154 & $81.4 \%$ & $0.50[0.28,0.73]$ & & \\
\hline Vinter 2011 & 18.1 & 3.1 & 37 & 15.8 & 3.1 & 35 & $18.6 \%$ & $0.73[0.26,1.21]$ & & \\
\hline Total $(95 \% \mathrm{Cl})$ & & & 187 & & & 189 & $100.0 \%$ & $0.55[0.34,0.75]$ & & \\
\hline \multicolumn{9}{|c|}{$\begin{array}{l}\text { Heterogeneity: } \text { Tau }^{2}=0.00 ; \mathrm{Chi}^{2}=0.72, \mathrm{df}=1(\mathrm{P}=0.40) \mathrm{I}_{1}^{2}=0 \% \\
\text { Test for overall effect: } Z=5.20(P<0.00001)\end{array}$} & $\begin{array}{lll}-2 & -1 & 0 \\
\text { Favours Control }\end{array}$ & $\begin{array}{cc}1 & 2 \\
\text { Favours Intervention }\end{array}$ \\
\hline
\end{tabular}

\section{MET $\mathrm{m} / \mathrm{wk}$, Metabolic Equivalent minutes per week; Steps, Steps per day; $\mathrm{VO}_{2}$ max, measure of physical fitness (oxygen used during exercise)}

Fig. 3 Meta-analysis of effect of interventions on physical activity outcomes

Secondary physical activity outcome $\mathrm{VO}_{2}$ max measures of physical fitness

Two studies compared $\mathrm{VO}_{2}$ max to measure the amount of oxygen used during exercise in order to assess physical fitness compared to control at follow up (Fig. 3). The studies were homogenous $\left(x^{2}=0.72\right.$, d.f. $\left.=1[P=0.40], \mathrm{I}^{2}=0 \%\right)$ and demonstrated significantly greater physical fitness in the intervention 
group compared to the control group (SMD 0.55 $[0.34,0.75], \mathrm{Z}=5.20 \mathrm{P}=<0.001)$.

\section{Other physical activity interventions}

Six additional trials that were not included in the metaanalyses due to insufficient data and different outcome measures reported varying intervention effects at follow up. Five of these studies reported an increase in physical activity or physical fitness for women in the intervention group compared to control [52, 53, 56, 64, 65]. Women who received diet and physical activity counselling increased their median weekly leisure time physical activity by $15 \mathrm{~min}$ (95\% [C1 $1-29 \mathrm{~min}$ ] while the physical activity of women in the control group remained unchanged ( $P=0.17$ unadjusted) [52]. Furthermore, one home based intervention using a stationary bicycle, found that women in the intervention group improved their aerobic fitness by increasing the test time taken to reach target heart rate of $150 \mathrm{bpm}(+48.0 ; P=0.019)$ compared to the control group [64]. Similarly, another home based intervention found a trend towards increased fitness following the intervention (indicated by higher cycling power output $\left.75 \% \mathrm{HR}_{\max }\right)(P=0.064,57)$ compared to the control. A supervised exercise programme consisting of treadmill walking and resistance training found that the proportion of women reporting regular exercise training in late pregnancy was significantly higher in the exercise group than in the control group: 77 and 23\% respectively $(P<0.01,66)$. However, one study that consisted of two intervention groups (passive consisting of brochure and physical activity advice; active group consisting of the same but included active counselling) found physical activity significantly decreased from first trimester to the third trimester by 0.62 in the control group, by 0.33 in the active group and by 0.09 in the passive group $(p=0.002,52)$.

\section{Effect on health outcomes}

Reductions in the incidence of GDM [52, 63], GWG [52, 55,59 ] and the number of new-borns with a birth weight of $>4000 \mathrm{~g}$ was significantly lower in the intervention group [63] compared to controls.

\section{Behaviour change techniques}

\section{Presence of BCTs}

A total of 19 different BCTs were applied within the 19 intervention studies, ranging between 1 and 10 in each study (Table 2). 'Self-monitoring of behaviour' and 'Instruction on how to perform the behaviour' were the most frequently described across the interventions and were identified in 13 out of the 19 studies (76.5\%). Information about health consequences was used in 8 out of the 19 interventions (47.1\%) and 'social support (unspecified)' was used in 7 out of the 17 interventions
Table 3 Frequencies of behaviour change techniques used in the interventions

\begin{tabular}{|c|c|c|c|c|}
\hline Groups & $\mathrm{BCT}$ & Number & Percent & $\begin{array}{l}\text { Average \# of time } \\
B C T \text { is used within } \\
\text { each intervention }{ }^{\text {a }}\end{array}$ \\
\hline \multirow[t]{6}{*}{$\begin{array}{l}\text { Goals and } \\
\text { planning }\end{array}$} & $\begin{array}{l}1.1 \text { Goal setting } \\
\text { (behaviour) }\end{array}$ & 4 & 23.5 & 6 \\
\hline & $\begin{array}{l}\text { 1.2 Problem } \\
\text { solving }\end{array}$ & 6 & 35.3 & 8.5 \\
\hline & $\begin{array}{l}1.3 \text { Goal setting } \\
\text { outcome }\end{array}$ & 7 & 41.2 & 4.3 \\
\hline & $\begin{array}{l}\text { 1.4 Action } \\
\text { Planning }\end{array}$ & 1 & 5.9 & 1 \\
\hline & $\begin{array}{l}1.5 \text { Review } \\
\text { behavioural } \\
\text { goals }\end{array}$ & 1 & 5.9 & 4 \\
\hline & $\begin{array}{l}\text { 1.7 Review } \\
\text { outcome goals }\end{array}$ & 1 & 5.9 & 5.5 \\
\hline \multirow[t]{3}{*}{$\begin{array}{l}\text { Feedback and } \\
\text { monitoring }\end{array}$} & $\begin{array}{l}2.2 \text { Feedback } \\
\text { on behaviour }\end{array}$ & 3 & 17.6 & 12.3 \\
\hline & $\begin{array}{l}2.3 \text { Self- } \\
\text { monitoring of } \\
\text { behaviour }\end{array}$ & 13 & 76.5 & 5.5 \\
\hline & $\begin{array}{l}2.4 \text { Self- } \\
\text { monitoring of } \\
\text { outcome of } \\
\text { behaviour }\end{array}$ & 2 & 11.8 & 1 \\
\hline Social support & $\begin{array}{l}3.1 \text { Social } \\
\text { Support } \\
\text { (Unspecified) }\end{array}$ & 7 & 41.2 & 11.1 \\
\hline $\begin{array}{l}\text { Shaping } \\
\text { Knowledge }\end{array}$ & $\begin{array}{l}4.1 \text { Instruction } \\
\text { on how to } \\
\text { perform } \\
\text { behaviour }\end{array}$ & 13 & 76.5 & 9.1 \\
\hline $\begin{array}{l}\text { Natural } \\
\text { consequences }\end{array}$ & $\begin{array}{l}5.1 \text { Information } \\
\text { about health } \\
\text { consequence }\end{array}$ & 8 & 47.1 & 1.6 \\
\hline \multirow[t]{2}{*}{$\begin{array}{l}\text { Comparison } \\
\text { of behaviour }\end{array}$} & $\begin{array}{l}6.1 \\
\text { Demonstration } \\
\text { of the } \\
\text { behaviour }\end{array}$ & 2 & 11.8 & 2 \\
\hline & $\begin{array}{l}6.2 \text { Social } \\
\text { comparison }\end{array}$ & 1 & 5.9 & 8 \\
\hline Associations & $\begin{array}{l}\text { 7.1 Prompt and } \\
\text { cues }\end{array}$ & 1 & 5.9 & 1 \\
\hline \multirow[t]{3}{*}{$\begin{array}{l}\text { Repetition } \\
\text { and } \\
\text { substitution }\end{array}$} & $\begin{array}{l}8.1 \text { Behavioural } \\
\text { practice/ } \\
\text { rehearsal }\end{array}$ & 6 & 35.3 & 21 \\
\hline & $\begin{array}{l}8.2 \text { Behaviour } \\
\text { substitution }\end{array}$ & 1 & 5.9 & 1 \\
\hline & $\begin{array}{l}8.3 \text { Habit } \\
\text { formation }\end{array}$ & 1 & 5.9 & 1 \\
\hline Antecedents & $\begin{array}{l}12.5 \text { Adding } \\
\text { objects to the } \\
\text { environment }\end{array}$ & 3 & 17.6 & 1 \\
\hline
\end{tabular}

$B C T$ behaviour change technique

a estimated number of times a BCT was potentially implemented based on intervention description in each study and by calculating an average for each BCT 
(41.2\%), an average 11.1 times within each intervention (Table 3). 'Social support (unspecified)' and 'Instruction on how to perform the behaviour' were identified in one comparator group which consisted of once-weekly sessions of relaxation, respiratory exercises and light stretching and focus group discussions concerning maternity [57]. Interrater reliability was calculated by a chance-corrected kappa $(k=0.65)$ indicating substantial agreement.

\section{Number of BCTs and effect size}

Subgroup analysis of which BCTs were associated with changes in physical activity outcome measures was not possible due to the small number of interventions included in the meta-analyses. The relationship between the total number of BCTs coded within an intervention and its effect size was found to be non-significant for MET $(r=0.20, p=0.63)$ and for steps per day $(r=0.89$, $p=0.31$ ). Pearson's $\mathrm{r}$ correlation coefficient was not calculated for $\mathrm{VO}_{2}$ max or for the other six studies not included in the meta-analyses due to insufficient data.

\section{Discussion}

The aim of this review was to identify and summarise the evidence for the effectiveness of physical activity interventions for pregnant women with overweight and obesity on physical activity levels. Furthermore, it set out to identify which BCTs are used in these physical activity interventions. Following a systematic screening process, 19 physical activity intervention studies were included. Due to the variation of physical activity outcomes, 13 studies were included in the meta-analyses. Three small separate meta-analyses found a positive effect on MET minutes per week and $\mathrm{VO}_{2}$ max for improving physical activity during pregnancy. As described by Currie et al. 2013, physical activity tends to decrease gradually throughout pregnancy, therefore any outcome that demonstrates greater physical activity than control is deemed to be a desirable outcome [26]. Thus, the results of this review suggest that physical activity interventions are to some extent effective at increasing physical activity levels for women with overweight and obesity. However, these results should be viewed with caution as the pooled data came from studies that were highly heterogeneous. Despite physical activity reducing as pregnancy progresses due to the physical impediments experienced by women in the third trimester [69], some of the studies in this review established some positive physical activity results including an increase in physical fitness and a slight reduction in the incidence of GDM [52, 56, 64]. However, these results should also be approached as tentative due to small number of studies and a lack of available data.

Thirteen studies included in the three small separate meta-analyses found a main effect on physical activity outcomes for MET minutes per week and $\mathrm{VO}_{2}$ max but not for steps per day which suggests that some physical activity interventions could be a beneficial strategy for improving physical activity during pregnancy. Additionally, five other studies (not included in the meta-analysis) reported an increase in physical activity or physical fitness for women in the intervention group compared to control. As physical activity guidelines recommend participation in moderate intensity activity on 'most days' [8], this is a positive finding regarding the efficacy of these physical activity interventions. However, the low number of studies and the inclusion of three pilot trials suggest that caution should be applied when interpreting these results. The wide range of physical activity measures used within the interventions reviewed creates difficulty for researchers and health care professionals trying to draw conclusions. For interventions that include a self-report measure of physical activity, social desirability bias may have led to women over reporting their physical activity levels. Although the majority of self-report questionnaires were based on valid and reliable measures, objective measures such as accelerometers have demonstrated a higher degree of reproducibility and validity for quantifying duration and intensity of physical activity [70, 71].

In the current review, the most commonly used BCT categories within the interventions were 'goals and planning', 'feedback and monitoring', 'social support', 'shaping knowledge' and 'natural consequences'. Other studies that have used the BCTs taxonomy to code lifestyle interventions in pregnancy have also found that categories such as 'goals and planning' and 'feedback and monitoring' were the most frequently used $[31,72,73]$. In this review, 'selfmonitoring of behaviour' (using items such as diaries or workbooks to monitor physical activity) and instruction on how to perform the behaviour' (providing participants with descriptions for particular exercises) emerged as the most frequently used BCTs across the interventions. Interventions which included these BCTs showed some positive effects but further research is required to examine the link between BCTs and intervention effectiveness. Research involving adults with overweight and obesity, also identified 'self-monitoring of behaviour' as a common $\mathrm{BCT}$ in physical activity interventions [74]. Furthermore, a review examining the use of pedometers to increase physical activity, found significant increases in physical activity in an adult population [75]. In pregnancy, women with overweight and obesity have indicated that pedometers and step counts could help with self-monitoring [76] with pedometers being found as an acceptable form of selfmonitoring [77]. Therefore, based on the results from this review and previous research, future interventions should include some component of self-monitoring in order to improve physical activity levels for pregnant women with overweight and obesity. While the BCTs used to promote physical activity in this review correspond closely to those 
found in previous antenatal interventions [31, 72], the identification of 'social support' is new to this pregnant population with overweight and obesity, with other systematic reviews of antenatal interventions failing to identify this BCT. Previous research has identified 'social influences' as an enabler to physical activity for women with overweight and obesity [76]. Furthermore, another study which investigated women's experiences of pregnancy found that physically active women faced some criticism from family members about their active lifestyles [78]. Thus, future interventions need to take into account the woman's social support network, to include family, friends and other pregnant women in these antenatal interventions. As previously found, this result highlights the importance of selecting appropriate BCTs for each population and not assuming all BCTs will be equally effective.

\section{Strengths and limitations}

This systematic review was comprehensive in its scope and search and was conducted in accordance with the PRISMA (preferred reporting items for systematic reviews and meta-analysis) statement [41]. A strength of this study was the use of an established instrument (BCTTv1) to systematically code the presence of BCTs in physical activity interventions for pregnant women with overweight and obesity.

The main limitations of this review stem from the inadequate reporting of physical activity data and poor intervention designs. Large differences in the type of activity measured, along with self-report measures highlights a limitation of the literature to date, making comparisons challenging. Also the use of physical fitness as a secondary outcome can be difficult to interpret. The studies lacked sufficient data to calculate pooled effect sizes for all physical activity outcome measures. Furthermore, while publication bias was not detected or performed for all outcomes, the majority of studies were of high risk of bias. Due to the small number of studies included in the metaanalysis and the high degree of heterogeneity, caution must be applied when generalising these findings. Therefore, the evidence base is weak and calls for more robust studies. Future research using robust high quality studies will foster better data to inform policy and practice.

The majority of interventions were based in a clinical setting which may have impacted intervention effectiveness. Furthermore, physical activity data were assessed using the last measure before birth (between 28 and 35 weeks' gestation) thus reducing comparability between studies with follow up ranging from 8 weeks' gestation to 12 months postpartum. Also, there were differences in the delivery modes and person, the intensity of the interventions and how active the women were prior to the intervention which may have also played a role in intervention effectiveness (and the BCTs used). As pregnancy progresses women tend to become less active [79], thus, future research is required to assess trimester (stage of pregnancy) and whether this impacts intervention effectiveness and the BCT employed.

Results from this review can be considered exploratory as no conclusions regarding the potential relationship between intervention content and effectiveness can be made. This was due to the paucity of intervention studies. A higher number of RCT studies of physical activity interventions for women with overweight and obesity during pregnancy are needed to draw firm conclusions. Many studies failed to provide adequate information on intervention content. As described by others, studies do not always provide adequate intervention content [80]. Not all studies had associated methods or protocol papers available making it possible that other BCTs were used but not coded. This, however, is a common problem conducting reviews such as these [28, 34, 81]. Furthermore, correlation of BCTs and outcomes has previously been identified as a methodological weakness [82]. It is difficult to know if routine antenatal care provided a BCT or not. In order to reliably identify the BCTs associated with physical activity for women with overweight and obesity, control groups identified as routine care should be described in intervention reports and coded for BCTs. Furthermore, as one control group contained BCTs, this creates a potential source of bias affecting the reliability of the data. Fidelity was poorly reported so it was impossible to determine if BCTs were delivered or received as intended.

Some of the BCT definitions were difficult to interpret, in particular 'Information about health consequence'. This definition was not explicit about whether 'health consequences' related to the positive or negative health outcomes of performing or not preforming the behaviour, respectively. Therefore, after detailed discussion 'Information about health consequence' was coded for both. Furthermore, intervention components such as free gym membership and swimming pool vouchers were used within two intervention studies $[52,55]$ and were not coded as BCTs; however these components could have an impact on behaviour change. In addition, contextual factors shape interventions and, therefore can influence how BCTs are delivered. Context can include individuals, teams, organisational structures and cultures, resources, leadership styles and relationships [83, 84].

Future interventions need to clearly define and report the behavioural outcome measure for physical activity such as core outcome sets for physical activity in pregnancy $[85,86]$. Furthermore, future intervention should follow TIdieR guidelines for reporting intervention content [87]. Moreover, interventions need to provide more transparent and comprehensive descriptions of $\mathrm{BCTs}$ 
used, and should include detail of context, fidelity, dose and clarity regarding the theory used within the intervention. Improved intervention description including the use of recognised and standardised taxonomies would increase ability to assess the BCTs and to examine the relationship between technique usage and change in physical activity. Despite these limitations, it is important to conduct such reviews enabling researchers to describe and analyse in detail the content of interventions, aiding the accuracy and communication required to build a cumulative evidence base [88].

\section{Conclusion}

The meta-analysis and narrative description of the included studies in this review revealed an increase in physical activity or physical fitness for pregnant women with overweight and obesity. A range of BCTs that could be used to help improve physical activity levels during pregnancy were identified, including: 'goals and planning', 'feedback and monitoring' and 'shaping knowledge' with 'social support' being newly identified for this population. Given the importance of physical activity to many subsequent outcomes in pregnancy, an explicit theoretical basis is needed for intervention development. Furthermore, interventions need to not only report the presence and frequency of BCTs but also the intensity and quality in which they are delivered or implemented. As 'social support' was identified within this review for a pregnant population with overweight or obesity future interventions need to take into account woman's social support networks, to include family and friends. These conclusions are tentative because of the high risk of bias of the included studies. Therefore, future studies should consider physical activity outcome carefully so that studies can be meaningfully compared. Intervention developers need to use recognised and standardised taxonomies to describe intervention content. To enable us to identify which BCTs are most effective for physical activity interventions with pregnant women with who are overweight and obese.

\section{Supplementary information}

Supplementary information accompanies this paper at https://doi.org/10. 1186/s12966-019-0859-5.

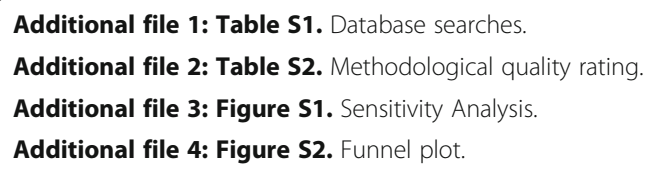

\section{Abbreviations}

BCT: Behaviour change technique; GDM: Gestational diabetes mellitus; MET: Metabolic equivalent; PA: Physical activity; RCT: Randomised controlled trial; $\mathrm{VO}_{2}$ max: Maximal oxygen uptake

\section{Acknowledgments}

Not applicable

\section{Author's contribution}

$C F, P K, M B, E O$ and FMA conceived and designed the study. CF, PK, MB, EO and FMA developed the review protocol and $C F$ registered the protocol with PROSPERO. CF conducted searches; CF, PK, MB, EO and FMA carried out screening. CF and MF carried out BCT coding for included articles. CF completed the analysis. CF wrote first draft of the paper. All authors contributed to successive drafts. All authors read and approved the final manuscript

\section{Funding}

CF was funded by the Health Research Board SPHeRE/2013/1 for this work. The Health Research Board (HRB) supports excellent research that improves people's health, patient care and health service delivery. The HRB aims to ensure that new knowledge is created and then used in policy and practice. In doing so, the HRB supports health system innovation and creates new enterprise opportunities.

\section{Availability of data and materials}

All data generated during this study are included in this published article [and its supplementary information files].

Ethics approval and consent to participate

Not applicable

\section{Consent for publication}

Not applicable

\section{Competing interests}

The authors declare that they have no competing interests.

\section{Author details}

${ }^{1}$ School of Public Health, University College Cork, Cork, Ireland. ${ }^{2}$ Health Behaviour Change Research Group, School of Psychology, National University of Ireland, Galway, Ireland. ${ }^{3}$ Centre for Maternal and Child Health Research, School of Health Sciences, City, University of London, London, United Kingdom. ${ }^{4}$ Perinatal Research Centre, School of Medicine, University College Dublin, National Maternity Hospital, Dublin, Ireland.

Received: 19 June 2019 Accepted: 10 October 2019

Published online: 01 November 2019

References

1. Centre for Public Health Excellence at Nice National Collaborating Centre for Primary C. National Institute for Health and Clinical Excellence: Guidance. Obesity: The Prevention, Identification, Assessment and Management of Overweight and Obesity in Adults and Children. London: National Institute for Health and Clinical Excellence (UK); 2006.

2. Campbell F, Johnson M, Messina J, Guillaume L, Goyder E. Behavioural interventions for weight management in pregnancy: a systematic review of quantitative and qualitative data. BMC Public Health. 2011;11(1):491.

3. Marchi J, Berg M, Dencker A, Olander EK, Begley C. Risks associated with obesity in pregnancy, for the mother and baby: a systematic review of reviews. Obes Rev. 2015;16(8):621-38.

4. Hinton PS, Olson CM. Predictors of pregnancy-associated change in physical activity in a rural White population. Matern Child Health J. 2001;5(1):7.

5. Gore SA, Brown DM, West DS. The role of postpartum weight retention in obesity among women: a review of the evidence. Ann Behav Med. 2003; 26(2):149-59.

6. The American College of Obstretricians and Gynecologists. Physical Activity and Exercise During Pregnancy and the Postpartum Period. https://www. acog.org/-/media/Committee-Opinions/Committee-on-Obstetric-Practice/ co736.pdf?dmc=1\&ts=20190222T1814547421. Update May 2015.

7. Artal R, O'Toole M. Guidelines of the American College of Obstetricians and Gynecologists for exercise during pregnancy and the postpartum period. $\mathrm{Br}$ J Sports Med. 2003;37(1):6-12.

8. RCOG CoOaG. Exercise in Pregnancy Statement 4. 2006; https://www.rcog. org.uk/globalassets/documents/guidelines/statements/statement-no-4.pdf. 
9. Nascimento SL, Surita FG, Cecatti JG. Physical exercise during pregnancy: a systematic review. Curr Opin Obstet Gynecol. 2012;24(6):387-94.

10. Evenson KR, Wen F. National trends in self-reported physical activity and sedentary behaviors among pregnant women: NHANES 1999-2006. Prev Med. 2010;50(3):123-8.

11. Walsh JM, McGowan C, Byrne J, McAuliffe FM. Prevalence of physical activity among healthy pregnant women in Ireland. Int J Gynaecol Obstet. 2011; 114(2):154-5.

12. RCOG. Recreational exercise and pregnancy: information for you. 2006. https://www.rcog.org.uk/globalassets/documents/patients/patientinformation-leaflets/pregnancy/recreational-exercise-and-pregnancy.pdf. [cited 201621 August]

13. Society of Obstetricians and Gynaecologists of Canada S, Canadian Society for Exercise Physiology C. Clinical Practice Guidelines: Exercise in pregnancy and the postpartum period 2003. https://sogc.org/wp-content/uploads/2 013/01/129E-JCPG-June03.pdf]. [cited 201619 August]

14. HSE/ICGP. Healthy Weight Management Guidelines Before, During \& After Pregnancy. https:/www.hse.ie/eng/health/child/healthyeating/ pregnancyguidelines.pdf. 2013

15. Health Service Executive. Obesity and Pregnancy Clinical Practice Guideline. 2013;Accessed 2016

16. Evenson KR, Savitz DA, Huston SL. Leisure-time physical activity among pregnant women in the US. Paediatr Perinat Epidemiol. 2004:18(6):400-7.

17. O'Keeffe LM, Dahly DL, Murphy M, Greene RA, Harrington JM, Corcoran P, et al. Positive lifestyle changes around the time of pregnancy: a crosssectional study. BMJ Open. 2016;6(5)::010233.

18. Obstetricians ACo, Gynecologists. ACOG Committee opinion no. 549: obesity in pregnancy. Obstet Gynecol. 2013;121(1):213.

19. Jepson RG, Harris FM, Platt S, Tannahill C. The effectiveness of interventions to change six health behaviours: a review of reviews. BMC Public Health 2010;10(1):538.

20. Michie S, Richardson M, Johnston M, Abraham C, Francis J, Hardeman W The behavior change technique taxonomy $(\mathrm{V} 1)$ of 93 hierarchically clustered techniques: building an international consensus for the reporting of behavior change interventions. Ann Behav Med. 2013;46:81-95.

21. Asbee SM, Jenkins TR, Butler JR, White J, Elliot M, Rutledge A. Preventing excessive weight gain during pregnancy through dietary and lifestyle counseling: a randomized controlled trial. Obstet Gynecol. 2009:113(2, Part 1):305-12.

22. Sui Z, Grivell RM, Dodd JM. Antenatal exercise to improve outcomes in overweight or obese women: a systematic review. Acta Obstet Gynecol Scand. 2012;91(5):538-45.

23. Ronnberg AK, Nilsson K. Interventions during pregnancy to reduce excessive gestational weight gain: a systematic review assessing current clinical evidence using the grading of recommendations, assessment, development and evaluation (GRADE) system. BJOG. 2010;117(11):1327-34.

24. Williams SL, French DP. What are the most effective intervention techniques for changing physical activity self-efficacy and physical activity behaviour - and are they the same? Health Educ Res. 2011;26(2):308-22.

25. Dodd JM, Grivell RM, Crowther CA, Robinson JS. Antenatal interventions for overweight or obese pregnant women: a systematic review of randomised trials. BJOG. 2010;117(11):1316-26.

26. Currie S, Sinclair M, Murphy MH, Madden E, Dunwoody L, Liddle D. Reducing the decline in physical activity during pregnancy: a systematic review of behaviour change interventions. PLoS One. 2013;8(6):e66385.

27. Collins LM, Murphy SA, Nair VN, Strecher VJ. A strategy for optimizing and evaluating behavioral interventions. Ann Behav Med. 2005;30(1):65-73.

28. Dombrowski SU, Sniehotta FF, Avenell A, Johnston M, MacLennan G, Araújo-Soares V. Identifying active ingredients in complex behavioural interventions for obese adults with obesity-related co-morbidities or additional risk factors for co-morbidities: a systematic review. Health Psychol Rev. 2012;6(1):7-32.

29. Cradock KA, ÓLaighin G, Finucane FM, Gainforth HL, Quinlan LR, Ginis KAM. Behaviour change techniques targeting both diet and physical activity in type 2 diabetes: a systematic review and meta-analysis. Int J Behav Nutr Phys Act. 2017;14(1):18

30. Skouteris H, Morris H, Nagle C, Nankervis A. Behavior modification techniques used to prevent gestational diabetes: a systematic review of the literature. Curr Diab Rep. 2014;14(4):480.

31. Gardner B, Wardle J, Poston L, Croker H. Changing diet and physical activity to reduce gestational weight gain: a meta-analysis. Obes Rev. 2011;12(7): e602-20.
32. Thangaratinam S, Rogozińska E, Jolly K, Glinkowski S, Roseboom T, Tomlinson JW, et al. Effects of interventions in pregnancy on maternal weight and obstetric outcomes: meta-analysis of randomised evidence. BMJ. 2012;344:e2088.

33. Bain E, Crane M, Tieu J, Han S, Crowther CA, Middleton P. Diet and exercise interventions for preventing gestational diabetes mellitus. Cochrane database Syst Rev. 2015;(4):Cd010443. https://www.ncbi.nlm.nih.gov/ pubmed/25864059.

34. Fredrix M, McSharry J, Flannery C, Dinneen S, Byrne M. Goal-setting in diabetes self-management: a systematic review and meta-analysis examining content and effectiveness of goal-setting interventions. Psychol Health. 2018;33(8):955-77.

35. Olander EK, Fletcher H, Williams S, Atkinson L, Turner A, French DP. What are the most effective techniques in changing obese individuals' physical activity self-efficacy and behaviour: a systematic review and meta-analysis. Int J Behav Nutr Phys Act. 2013;10(29):1-15.

36. Lawlor DA, Chaturvedi N. Treatment and prevention of obesity -are there critical periods for intervention? Oxford University Press; 2006. https://www. ncbi.nlm.nih.gov/pubmed/16766539.

37. Jeffries K, Shub A, Walker SP, Hiscock R, Permezel M. Reducing excessive weight gain in pregnancy: a randomised controlled trial. Med J Aust. 2009; 191(8):429-33.

38. Polley BA, Wing R, Sims C. Randomized controlled trial to prevent excessive weight gain in pregnant women. Int J Obes Relat Metab Disord. 2002; 26(11):1494-502.

39. Hui AL, Ludwig S, Gardiner P, Sevenhuysen G, Murray R, Morris M, et al. Community-based exercise and dietary intervention during pregnancy: a pilot study. Can J Diabetes. 2006;30(2):1-7.

40. Phelan S, Phipps MG, Abrams B, Darroch F, Schaffner A, Wing RR. Randomized trial of a behavioral intervention to prevent excessive gestational weight gain: the fit for delivery study. Am J Clin Nutr. 2011;93(4):772-9.

41. Moher D, Liberati A, Tetzlaff J, Altman DG. Preferred reporting items for systematic reviews and meta-analyses: the PRISMA statement. Ann Intern Med. 2009:151(4):264-9.

42. Cohen J. A coefficient of agreement for nominal scales. Educ Psychol Meas. 1960;20(1):37-46.

43. Landis JR, Koch GG. The measurement of observer agreement for categorical data. Biometrics. 1977:159-74. https:/www.ncbi.nlm.nih.gov/pubmed/21310836.

44. Albrecht L, Archibald M, Arseneau D, Scott SD. Development of a checklist to assess the quality of reporting of knowledge translation interventions using the workgroup for intervention development and evaluation research (WIDER) recommendations. Implement Sci. 2013:8(1):52.

45. Green Ha. Cochrane handbook for systematic reviews of interventions https://training.cochrane.org/handbook. 2011.

46. Hozo SP, Djulbegovic B, Hozo I. Estimating the mean and variance from the median, range, and the size of a sample. BMC Med Res Methodol. 2005:5(1):13.

47. Egger M, Smith GD, Schneider M, Minder C. Bias in meta-analysis detected by a simple, graphical test. BMJ. 1997;315(7109):629-34.

48. Callaway LK, Colditz PB, Byrne NM, Lingwood BE, Rowlands IJ, Foxcroft K, et al. Prevention of gestational diabetes feasibility issues for an exercise intervention in obese pregnant women. Diabetes Care. 2010;33(7):1457-9.

49. Hawkins M, Hosker M, Marcus BH, Rosal MC, Braun B, Stanek EJ 3rd, et al. A pregnancy lifestyle intervention to prevent gestational diabetes risk factors in overweight Hispanic women: a feasibility randomized controlled trial. Diabet Med. 2015;32(1):108-15.

50. Kong KL, Campbell CG, Foster RC, Peterson AD, Lanningham-Foster L. A pilot walking program promotes moderate-intensity physical activity during pregnancy. Med Sci Sports Exerc. 2014;46(3):462-71.

51. Guelinckx I, Devlieger R, Mullie P, Vansant G. Effect of lifestyle intervention on dietary habits, physical activity, and gestational weight gain in obese pregnant women: a randomized controlled trial. Am J Clin Nutr. 2009;91 (2):373-80.

52. Koivusalo SB, Rono K, Klemetti MM, Roine RP, Lindstrom J, Erkkola M, et al. Gestational diabetes mellitus can be prevented by lifestyle intervention: the Finnish gestational diabetes prevention study (RADIEL): a randomized controlled trial. Diabetes Care. 2016;39(1):24-30.

53. Nascimento S, Surita F, Parpinelli M, Siani S, Pinto e Silva J. The effect of an antenatal physical exercise programme on maternal/perinatal outcomes and quality of life in overweight and obese pregnant women: a randomised clinical trial. BJOG. 2011;118(12):1455-63.

54. Oostdam N, Van Poppel M, Wouters M, Eekhoff E, Bekedam D, Kuchenbecker W, et al. No effect of the FitFor2 exercise programme on 
blood glucose, insulin sensitivity, and birthweight in pregnant women who were overweight and at risk for gestational diabetes: results of a randomised controlled trial. BJOG. 2012;119(9):1098-107.

55. Vinter CA, Jensen DM, Ovesen P, Beck-Nielsen H, Jorgensen JS. The LiP (lifestyle in pregnancy) study: a randomized controlled trial of lifestyle intervention in 360 obese pregnant women. Diabetes Care. 2011;34(12):2502-7.

56. Ong M, Guelfi K, Hunter T, Wallman K, Fournier P, Newnham J. Supervised home-based exercise may attenuate the decline of glucose tolerance in obese pregnant women. Diabetes Metab. 2009;35(5):418-21.

57. Santos IA, Stein R, Fuchs SC, Duncan BB, Ribeiro JP, Kroeff $L R$, et al. Aerobic exercise and submaximal functional capacity in overweight pregnant women: a randomized trial. Obstet Gynecol. 2005;106(2):243-9.

58. Kennelly MA, Ainscough K, Lindsay KL, O'Sullivan E, Gibney ER, McCarthy M, et al. Pregnancy exercise and nutrition with smartphone application support: a randomized controlled trial. Obstet Gynecol. 2018;131(5):818-26.

59. Van Horn L, Peaceman A, Kwasny M, Vincent E, Fought A, Josefson J, et al. Dietary approaches to stop hypertension diet and activity to limit gestational weight: maternal offspring Metabolics family intervention trial, a technology enhanced randomized trial. Am J Prev Med. 2018;55(5):603-14.

60. Dodd JM, Cramp C, Sui Z, Yelland LN, Deussen AR, Grivell RM, et al. The effects of antenatal dietary and lifestyle advice for women who are overweight or obese on maternal diet and physical activity: the LIMIT randomised trial. BMC Med. 2014;12(1):161.

61. Poston L, Bell R, Croker H, Flynn AC, Godfrey KM, Goff L, et al. Effect of a behavioural intervention in obese pregnant women (the UPBEAT study): a multicentre, randomised controlled trial. Lancet Diab Endocrinol. 2015;3(10):767-77.

62. Renault KM, Nørgaard K, Nilas L, Carlsen EM, Cortes D, Pryds O, et al. The Treatment of Obese Pregnant Women (TOP) study: a randomized controlled trial of the effect of physical activity intervention assessed by pedometer with or without dietary intervention in obese pregnant women. Am J Obstet Gynecol. 2014;210(2):134. e1-9.

63. Bruno R, Petrella E, Bertarini V, Pedrielli G, Neri I, Facchinetti F. Adherence to a lifestyle programme in overweight/obese pregnant women and effect on gestational diabetes mellitus: a randomized controlled trial. Maternal \& Child Nutrition. 2017:13:e12333. https://doi.org/10.1111/mcn.12333.

64. Seneviratne S, Jiang Y, Derraik J, McCowan L, Parry G, Biggs J, et al. Effects of antenatal exercise in overweight and obese pregnant women on maternal and perinatal outcomes: a randomised controlled trial. BJOG. 2016; 123(4):588-97.

65. Garnaes KK, Morkved S, Salvesen O, Moholdt T. Exercise training and weight gain in obese pregnant women: a randomized controlled trial (ETIP Trial) [with consumer summary]. PLoS Med. 2016;13(7):e1002079.

66. Szmeja MA, Cramp C, Grivell RM, Deussen AR, Yelland LN, Dodd JM. Use of a DVD to provide dietary and lifestyle information to pregnant women who are overweight or obese: a nested randomised trial. BMC Pregnancy Childbirth. 2014;14(1):409

67. Medicine ACOS. ACSM's guidelines for exercise testing and prescription: Lippincott Williams \& Wilkins; 2013. http://antoinedl.com/fichiers/public/ ACSM-guidelines-2014.pdf.

68. Ainsworth BE, Haskell WL, Whitt MC, Irwin ML, Swartz AM, Strath SJ, et al. Compendium of physical activities: an update of activity codes and MET intensities. Med Sci Sports Exer. 2000:32(9 Suppl):S498-504.

69. Poudevigne MS, O'Connor PJ. A review of physical activity patterns in pregnant women and their relationship to psychological health. Sports Med. 2006;36(1):19-38.

70. Bell R, Tennant PWG, MCParlin C, Pearce MS, Adamson AJ, Rankin J, et al. Measuring physical activity in pregnancy: a comparison of accelerometry and self-completion questionnaires in overweight and obese women. Eur J Obstetr Gynecol Reprod Biol. 2013;170(1):90-5.

71. Corder K, Brage S, Ekelund U. Accelerometers and pedometers: methodology and clinical application. Curr Opin Clin Nutr Metab Care. 2007;10(5):597-603.

72. Hill B, Skouteris H, Fuller-Tyszkiewicz M. Interventions designed to limit gestational weight gain: a systematic review of theory and meta-analysis of intervention components. Obes Rev. 2013;14(6):435-50.

73. Soltani $H$, Arden M, Duxbury A, Fair F. An analysis of behaviour change techniques used in a sample of gestational weight management trials. J Pregnancy. 2016;2016:1085916.

74. Samdal GB, Eide GE, Barth T, Williams G, Meland E. Effective behaviour change techniques for physical activity and healthy eating in overweight and obese adults; systematic review and meta-regression analyses. Int J Behav Nutr Phys Act. 2017;14(1):42.

75. Bravata DM, Smith-Spangler C, Sundaram V, Gienger AL, Lin N, Lewis R, et al. Using pedometers to increase physical activity and improve health: a systematic review. JAMA. 2007;298(19):2296-304.

76. Flannery C, McHugh S, Anaba AE, Clifford E, O'Riordan M, Kenny LC, et al. Enablers and barriers to physical activity in overweight and obese pregnant women: an analysis informed by the theoretical domains framework and COM-B model. BMC Pregnancy Childbirth. 2018;18(1):178.

77. Renault K, Nørgaard K, Andreasen KR, Secher NJ, Nilas L. Physical activity during pregnancy in obese and normal-weight women as assessed by pedometer. Acta Obstet Gynecol Scand. 2010;89(7):956-61.

78. Atkinson L, Shaw RL, French DP. Is pregnancy a teachable moment for diet and physical activity behaviour change? An interpretative phenomenological analysis of the experiences of women during their first pregnancy. Br J Health Psychol. 2016;21(4):842-58.

79. Gaston A, Cramp A. Exercise during pregnancy: a review of patterns and determinants. J Sci Med Sport. 2011;14(4):299-305.

80. Riley BL, MacDonald J, Mansi O, Kothari A, Kurtz D, VonTettenborn LI, et al. Is reporting on interventions a weak link in understanding how and why they work? A preliminary exploration using community heart health exemplars. Implementation Sci. 2008:3(1):27.

81. Michie S, Abraham C, Whittington C, McAteer J, Gupta S. Effective techniques in healthy eating and physical activity interventions: a metaregression. Health Psychol. 2009;28(6):690-701.

82. Peters GJ, de Bruin M, Crutzen R. Everything should be as simple as possible, but no simpler: towards a protocol for accumulating evidence regarding the active content of health behaviour change interventions. Health Psychol Rev. 2015;9(1):1-14.

83. McCormack B, Kitson A, Harvey G, Rycroft-Malone J, Titchen A, Seers K. Getting evidence into practice: the meaning of 'context'. J Adv Nurs. 2002;38(1):94-104.

84. Wells M, Williams B, Treweek S, Coyle J, Taylor J. Intervention description is not enough: evidence from an in-depth multiple case study on the untold role and impact of context in randomised controlled trials of seven complex interventions. Trials. 2012;13(1):95.

85. Dadouch R, Faheim M, Juando-Prats C, Parsons J, D'Souza R. Development of a Core outcome set for studies on obesity in pregnant patients (COSSOPP): a study protocol. Trials. 2018;19(1):655.

86. Rogozinska E, Marlin N, Yang F, Dodd JM, Guelfi K, Teede H, et al. Variations in reporting of outcomes in randomized trials on diet and physical activity in pregnancy: a systematic review. J Obstet Gynaecol Res. 2017;43(7):1101-10

87. Hoffmann TC, Glasziou PP, Boutron I, Milne R, Perera R, Moher D, et al. Better reporting of interventions: template for intervention description and replication (TIDieR) checklist and guide. BMJ. 2014;348:91687.

88. Bishop FL, Fenge-Davies AL, Kirby S, Geraghty AWA. Context effects and behaviour change techniques in randomised trials: a systematic review using the example of trials to increase adherence to physical activity in musculoskeletal pain. Psychol Health. 2015;30(1):104-21.

\section{Publisher's Note}

Springer Nature remains neutral with regard to jurisdictional claims in published maps and institutional affiliations.

Ready to submit your research? Choose BMC and benefit from:

- fast, convenient online submission

- thorough peer review by experienced researchers in your field

- rapid publication on acceptance

- support for research data, including large and complex data types

- gold Open Access which fosters wider collaboration and increased citations

- maximum visibility for your research: over $100 \mathrm{M}$ website views per year

At $\mathrm{BMC}$, research is always in progress.

Learn more biomedcentral.com/submission 